\title{
The sialyl-glycolipid SSEA4 marks a subpopulation of chemotherapy resistant breast cancer cells with mesenchymal features
}

\author{
Andrea Aloia ${ }^{1,2^{*}}$, Evgeniya Petrova ${ }^{3}$, David Agorku$^{4}$, Annalisa Terranegra ${ }^{5}$, Alessandra Mingione $^{6}$, \\ Jean-Gabriel Judde ${ }^{7}$, Andreas Bosio ${ }^{4}$, Stefano Cairo ${ }^{7}$, Olaf Hardt ${ }^{4}$
}

From Breast Cancer Immunotherapy Symposium (BRECIS), part of the Sidra Symposia Series, held in partnership with the Society for Immunotherapy of Cancer

Doha, Qatar. 13-14 April 2015

Triple negative breast cancer (TNBC) is an aggressive breast cancer subtype associated with high risk of early relapse and metastasis [1]. At the moment chemotherapy remains the main option for systemic therapy of TNBC patients but complete remission occurs only in $20 \%$ of the patients [2]. In order to identify biomarker for chemotherapy-resistant TNBC cells, we performed a cell surface marker screen in 4 TNBC patient-derived xenograft (PDX) models that respond well to adriamycin/ cyclophosphamide-based $(\mathrm{A} / \mathrm{C})$ chemotherapy but fail to reach complete pathological response. We used multiparameter flow cytometry to screen the expression of 45 cell surface markers during the course of chemotherapy.

We identified the sialyl-glycolipid SSEA4 as a marker of chemotherapy-resistant cancer cells in all four models. In addition, 3 out of 4 TNBC PDXs showed higher percentage of SSEA4-positive cells compared to all A/C-sensitive TNBC PDXs analysed. Gene expression comparison between SSEA4-positive and SSEA4-negative tumor cells from 3 TNBC PDXs highlighted an overexpression of mesenchymal-associated genes and a deregulation of drug resistance pathway-associated genes and miRNAs in SSEA4+ breast cancer cells. In addition, high expression of ST3 beta-galactoside alpha-2,3-sialyltransferase 2 (ST3Gal2), the enzyme catalyzing the last step of SSEA4 synthesis, was found associated with poor outcome in ER-, PR- breast cancer patients treated with chemotherapy ( $\mathrm{p}<0.01$, HR 3.08).

Thus, we propose SSEA4 as a novel marker of mesenchymal and chemoresistant breast cancer cells, and ST3GAL2 expression as a predictive marker for chemoresistance associated with poor outcome in breast cancer patients.

\section{Authors' details}

${ }^{1}$ Institute for Molecular Health Sciences, ETH Zurich, Switzerland. ${ }^{2}$ Miltenyi Biotec GmbH, Bergisch Gladbach, Germany. ${ }^{3}$ R\&D, Xentech SAS, Institute Pasteur, Evry, France. ${ }^{4} \mathrm{R} \& \mathrm{D}$, Miltenyi Biotec $\mathrm{GmbH}$, Bergisch Gladbach, Germany. ${ }^{5}$ nvestigator Nutrigenetics, Sidra Medical and Research Center, Doha, Qatar. ${ }^{6}$ Department of Health Sciences, University of Milan, Milan, Italy. ${ }^{7}$ XenTech SAS, Evry, France.

Published: 14 August 2015

\section{References}

1. Irvin WJ Jr, Carey LA: What is triple-negative breast cancer? Eur J Cancer 2008, 44(18):2799-805

2. Keam B, Im SA, Kim HJ, Oh DY, Kim JH, Lee SH, Chie EK, Han W, Kim DW, Moon WK, Kim TY, Park IA, Noh DY, Heo DS, Ha SW, Bang YJ: BMC Prognostic impact of clinicopathologic parameters in stage II/III breast cancer treated with neoadjuvant docetaxel and doxorubicin chemotherapy: paradoxical features of the triple negative breast cancer. Cancer 2007, 7:203.

doi:10.1186/2051-1426-3-S1-01

Cite this article as: Aloia et al: The sialyl-glycolipid SSEA4 marks a subpopulation of chemotherapy resistant breast cancer cells with mesenchymal features. Journal for ImmunoTherapy of Cancer 2015 3(Suppl 1):01.

'Institute for Molecular Health Sciences, ETH Zurich, Switzerland

Full list of author information is available at the end of the article 